\title{
Snake Perturbations during Pellet injection and LHCD in the HL-1M Tokamak
}

\author{
Yi Liu Xiaoming Qiu, Yunbo Dong, YunzheZhong, Bingzhong Fu, Jiafu Dong Yong Liu
}

Southwestern Institute of Physics, Chengdu 610041, China

\author{
Email Address: yiliu@swip.ac.cn
}

\begin{abstract}
Excitation of snake perturbations has been observed in the core region of pellet-fuelled HL-1M plasmas when the pellets cross surface with $\mathrm{q}$ value 1 . Through measurements of plasma $\mathrm{q}$ profile by means of multi-exposures with CCD camera during pellet ablation, and investigation on pellet ablation process, possible mechanisms for the formation of snake oscillation are discussed. In addition, a large, long-lived snake-like oscillation is frequently observed in lower hybrid current driven discharge in which the sawtooth has been stabilized at early times. There is evidence that such a perturbation is due to impurity accumulation during sawtooth-stabilization, and the good performance with peaking profiles after LHCD is limited by magnetohydrodynamic (MHD) instabilities including sawtooth and snake activities in HL-1M plasma.
\end{abstract}

\section{Introduction}

Pellet injection experiments have been widely carried out with various toroidal plasmas, peaked density profile and improved confinement are frequently observed, and some interesting phenomena have also been found. One of them is the long-lived $m=1 / n=1$ oscillation observed on soft X-ray emission and other diagnostics after pellet injection in JET [1], JT-60 [2], and TORE SUPRA [3], which has been called the snake oscillation. The formation of snake reflects some important information about the changes in local plasma parameter, and how the plasma responds to the perturbation caused by pellet injection, it can also be used as a probe for the $\mathrm{q}=1$ surface. These provide the basic motivation for a better understanding of the snake process and its relationship with plasma parameters.

Snakes are observed in HL-1M following the injection of high speed solid hydrogen pellets during ohmic discharges, they are also observed in LHCD plasmas of HL-1M at the end or later phase of the suppression of sawtooth by LHCD. This paper will introduce the experimental observations on snake after pellet injection and during LHCD on HL-1M.

\section{Snake Perturbations in pellet fuelled plasma}

\subsection{Experimental results on excitation of snakes}

The HL-1M tokamak has circular cross section with major radius $\mathrm{R}=1.02 \mathrm{~m}$, minor radius $\mathrm{a}=0.26 \mathrm{~m}$. The experiment was performed with line-averaged electron density $\mathrm{n}_{\mathrm{e}}=0.3-3.6 \times 10^{19} \mathrm{~m}^{-3}$, central electron temperature $\mathrm{T}_{\mathrm{e}}(0) \approx 900 \mathrm{ev}$,plasma current $\mathrm{I}_{\mathrm{p}}$ in the range $100-200 \mathrm{kA}$, toroidal magnetic field in the range $2.0-2.6 \mathrm{~T}$ at $\mathrm{R}=1.02 \mathrm{~m}$. High speed hydrogen pellets with a size of $d_{P}=1.2 \mathrm{~mm}$ and with velocities in the range of 200 to $1100 \mathrm{~m} / \mathrm{s}$ have been injected into ohmically heated plasma in the HL-1M tokamak. One set of the SX array, a $H_{\alpha}$ emission array, and a CCD camera are located at same poloidal section with pellet injector to monitor the plasma radiation.

Excitation of large snake-like oscillation has been observed in the core region after injection of high-speed pellets into the HL-1M plasma. At moderated density $\left(\mathrm{n}_{\mathrm{e}}=1.5-3.0 \times 10^{19} \mathrm{~m}^{-3}\right)$ the pellets penetrated to and beyond the $\mathrm{q}=1$ surface. Figure 1 shows 
the temporal evolution of $\mathrm{H} \alpha$ emission signal along the pellet injection direction, the reduction in $\mathrm{H} \alpha$ emission as the pellet crosses $\mathrm{q}=1$ surface is clearly visible. The parameters for this shot (6163) before pellet injection are: $\mathrm{I}=150 \mathrm{kA}, \mathrm{B}=2.2 \mathrm{~T}, \mathrm{~T}_{\mathrm{e}}=0.93 \mathrm{kev}$ and $\mathrm{n}_{\mathrm{e}} \approx 2.2 \times 10^{19} \mathrm{~m}^{-3}$. Figure 2 gives time evolvement of $\mathrm{H} \alpha$ emission observed by multi-channel detectors, showing the pellet trajectory in the plasma. The ablation minimum determined the radius of $\mathrm{q}=1$ surface with $\mathrm{r}_{1}=4 \mathrm{~cm}$, which is similar to the values inferred from sawtooth inversion radius $\left(\mathrm{r}_{1}=3.5-4.0 \mathrm{~cm}\right)$ at the time just before pellet injection.

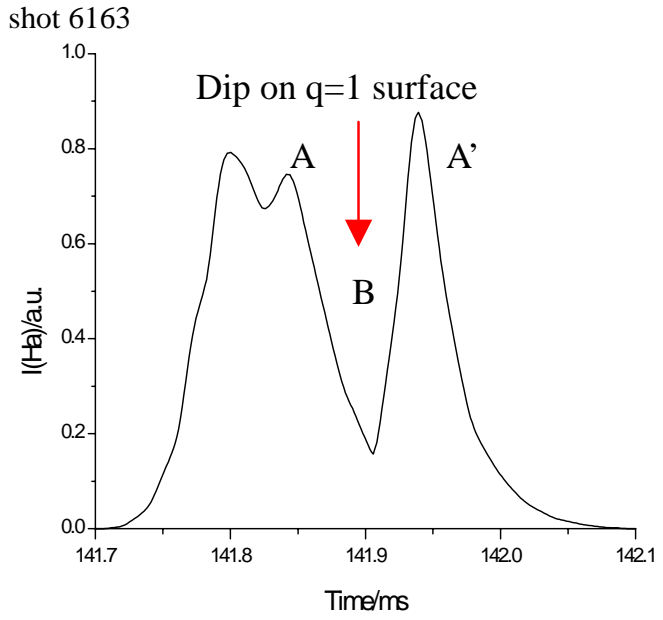

Figure 1. Temporal evolution of $\mathrm{H}_{\alpha}$ signal. The reduction in $\mathrm{H} \alpha$ emission as the pellet crosses $\mathrm{q}=1$ surface is clearly visible.

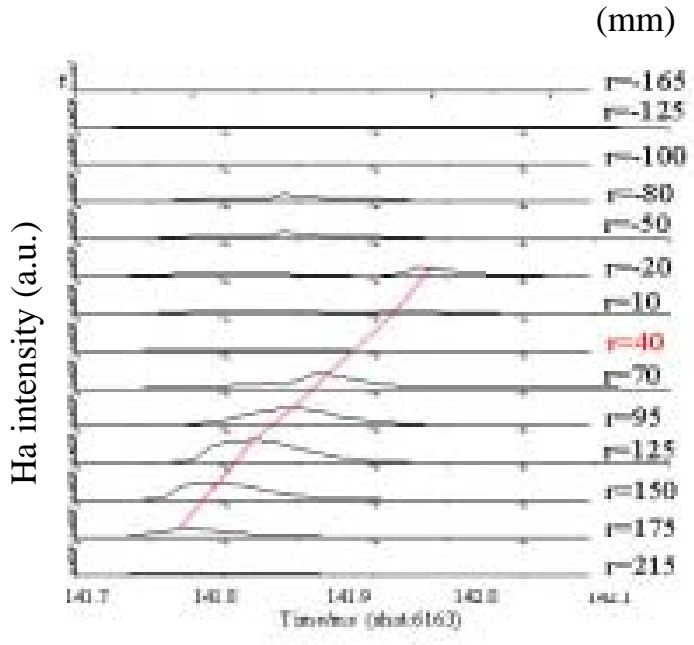

Figure 2. Evolvement of $\mathrm{H \alpha}$ emission observed by multi-channel detectors, showing the pellet trajectory in the plasma.

Strong bursts of snake oscillation frequently appear when the pellets cross the $q=1$ surface, Figure 3 shows the corresponding snake after pellet injection as seen by the soft X-ray camera, it has a minor radius $\left(\mathrm{r}_{\mathrm{s}}\right.$ ) of roughly $3 \mathrm{~cm}$ and has been found with an $\mathrm{m}=1$ topology by a comparison of the phase of the snake on all the diagnostics. The radial dimension and poloidal dimension of snake were determined from the time evolutions of the suitable soft $\mathrm{X}$-ray channels as $\ell_{r}=2.5 \mathrm{~cm}, \ell_{\theta}=3 \mathrm{~cm}$, respectively. Poloidally the snake rotates with a rotating frequency of $0.7 \mathrm{kHz}$ and the oscillation usually terminates after about ten msec. Based on SVD, we made a background subtraction to make the snake-like oscillation obvious in profile evolution. The subtraction is performed by reconstructing the signals, but discarding the largest singular value. The discarded part is related to the average shape of the profile, represented by the first eigenfunctions of space and time. Furthermore, we do a profile inversion, and the evolution of profile in time is plotted as figure 4 . It gives a clear image of the profile evolution showing a snake-like pattern.

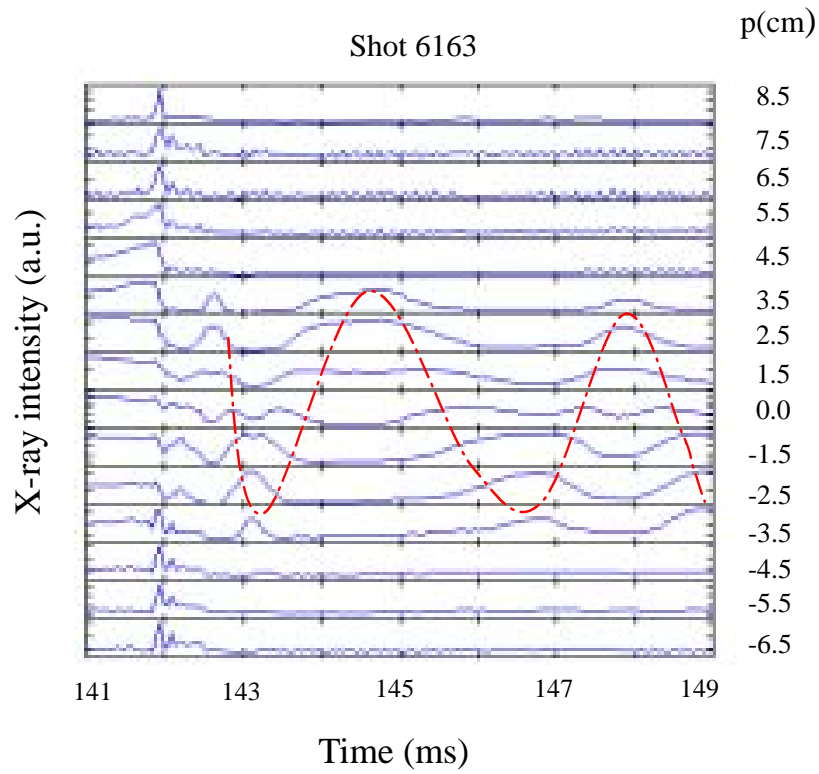

Figure 3. Time history of a pellet produced snake. It persists for nearly $10 \mathrm{~ms}$ after its production. 
In ohmic plasmas, under the condition of pellet having crossed the $\mathrm{q}=1$ surface, snakes are produced in about $60 \%$ of the cases. The probability of snake production becomes high when pellets penetrate deeper inside the $\mathrm{q}=1$ surface. On the other hand, when snake is produced, sawteeth very rarely appear. This is contrast to the observations in other tokamaks where the snake can co-exist with sawtooth and survive to the sawtooth crashes.

\subsection{Analysis of snake formation}

The result obtained on $\mathrm{HL}-1 \mathrm{M}$ is in broad agreement with previously reported data. Usually, snake is thought to be a result of an island formation due to the ablated particles flowing within the $\mathrm{q}=1$ resonant flux tube and producing a high density and lower temperature region [4]. On the other hand, there is small quantity of particles ablated near $\mathrm{q}=1$ rational surface because of the reduced reservoir of hot electrons available for ablating the pellet within the resonant flux tube as mentioned in section 2.1, while a large amount of particles are ablated inside the $\mathrm{q}=1$ surface (with a distance from $\mathrm{q}=1$ surface

of $\delta \approx v_{p_{\text {center }}} \times \Delta t \approx 16 \mathrm{~mm}, v_{p_{\text {center }}} \approx 360 \mathrm{~m} / \mathrm{s}$, as in figure 2) during the ablation process. Observation by the soft X-ray system also indicated the location of the snake oscillation is inside the $\mathrm{q}=1$ surface, which is determined from the $H_{\alpha}$ minimum, $\mathrm{r}_{s} / \mathrm{r}_{1} \approx 60-70 \%$. The

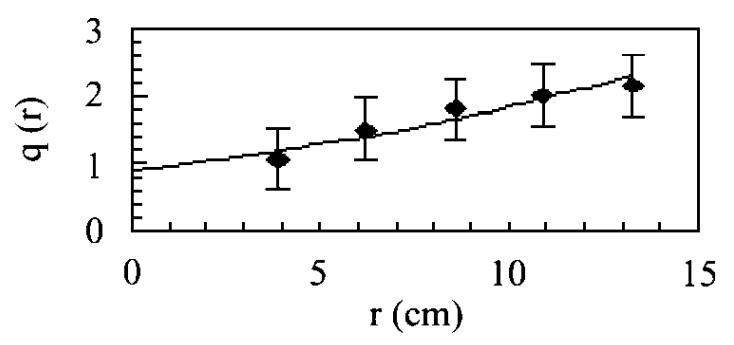

Figure 5. Profiles of the safety factor $q$ derived from the $H_{\alpha}$ striations during pellet iniection for shot 6163 .
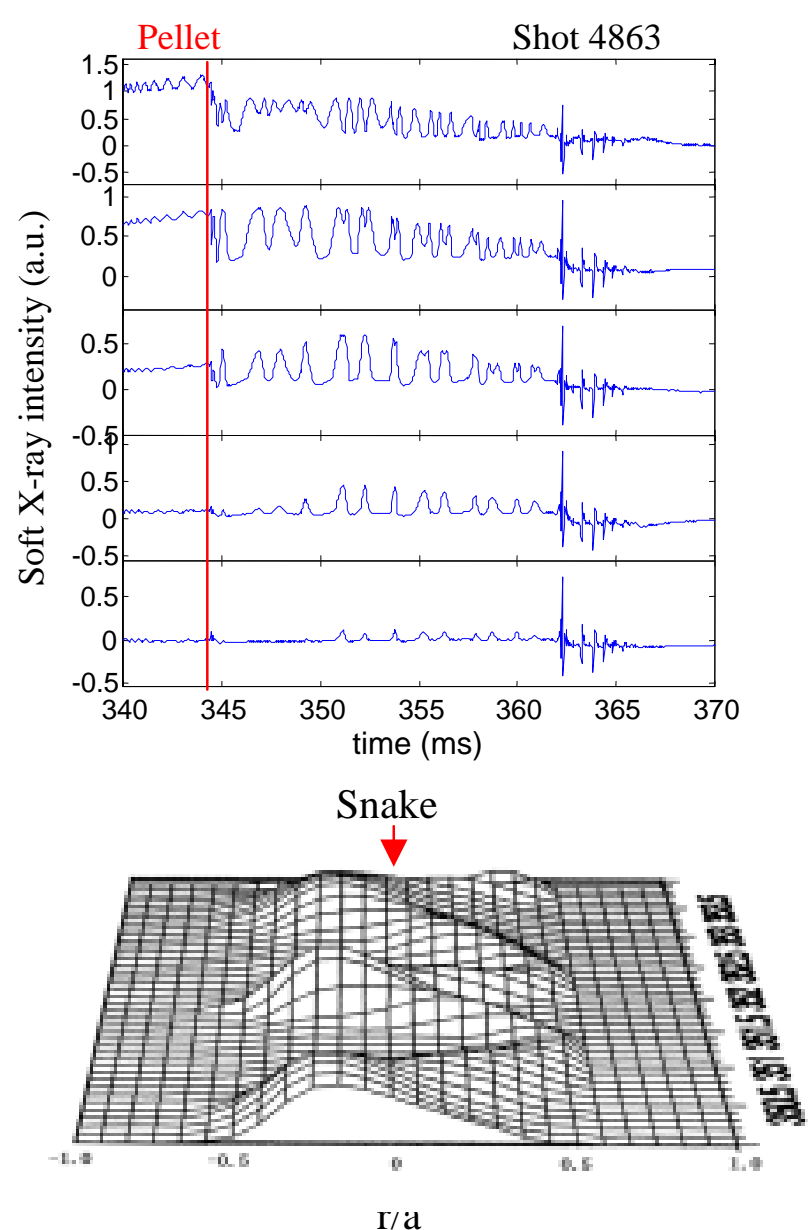

Figure 4. A hidden-line perspective plot of the reconstructed soft $\mathrm{X}$-ray emission, it shows a snake-like oscillation.

difference between $r_{s}=2.5-3 \mathrm{~cm}$ and $r_{1}=4.0-4.5 \mathrm{~cm}$ clearly indicated that the snake rotates on a surface well inside the $\mathrm{q}=1$ surface, this difference has also be observed in Toresupra.

By means of multi-exposures with a CCD camera during pellet ablation, the safety factor profile can be estimated by observing the orientation of the plasma tail originating from the pellet ablation region [5]. Figure 5 shows the calculated q profile using the inclination angle of the pellet ablation cloud, indicating the presence of a large plateau around $\mathrm{q}=1$ surface. This initial flat $\mathrm{q}$ profile gives the large shift in $\mathrm{r}_{1}$ possible, i.e., the $\mathrm{q}=1$ surface shifted inside during the transient phase. Typically a shift of $\Delta r_{1} / r_{1} \approx-\frac{1}{3}$ was observed in the experiments during snake oscillation. And a shift in $\mathrm{q}=1$ surface has also been observed in JET during a snake oscillation when there was a sawtooth 


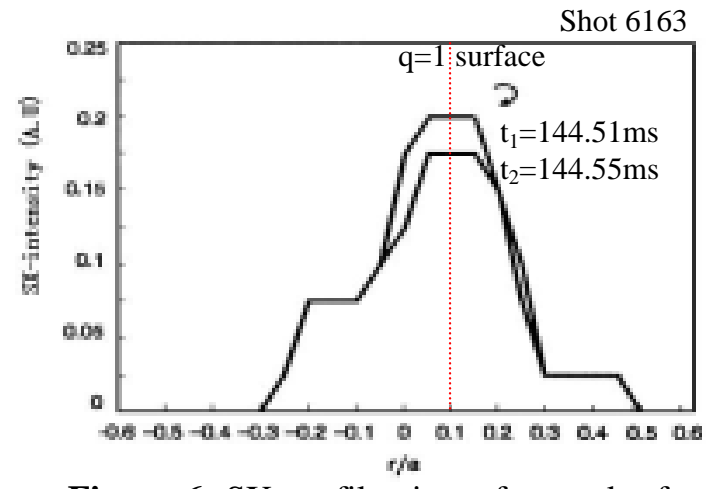

Figure 6. $\mathrm{SX}$ profiles just after snake formation at $\mathrm{t}_{1}=144.51 \mathrm{~ms}$ and $\mathrm{t}_{2}=144.55 \mathrm{~ms}$. The soft $\mathrm{x}$ ray intensity profile is peaked around $\mathrm{q}=1$ surface. collapse [1]. Near the shifted rational $q=1$ surface where a large amount of particles are deposited, there is a current depletion caused by the increased local resistivity. As a consequence, a magnetic island that traps particles released from the pellet is formed, producing a small region with high density. When the pressure inside the magnetic island is higher than that of the core plasma, it was found that the $\mathrm{m}=1$ mode can saturate [6]. In fact, from the equation for the linear growth rate of the $\mathrm{m}=1$ mode,

$\gamma=K \int_{0}^{r_{1}}\left(-2 \varepsilon^{2} r^{2} \frac{\partial P}{\partial r}+\varepsilon^{2} r B_{\theta}^{2}(3 q+1)(1-q) d r\right.$, where $\mathrm{r}_{1}$ is the position of the $\mathrm{q}=1$ surface, it is clear that an inverted pressure profile, i.e., $\mathrm{P}_{\text {center }}<\mathrm{P}_{\text {island }}$, can make the mode stable $(\gamma<0)$. Experimentally a peaking of the soft $\mathrm{x}$ ray emission around $\mathrm{q}=1$ surface is observed as shown in Figure 6 (shot 6163), and we approximate the pressure profile by the soft X-ray profile in our analysis as the SX intensity is roughly proportional to the electron pressure squared $\left(n_{e}^{2} T_{e}^{2}\right)$. A more complete analysis [7], which takes the pressure-driven current into account, $J_{p}(\sigma): J_{p}(\sigma)=\left(\frac{\delta \beta_{p}^{*}}{\lambda}\right) \frac{d F}{d \sigma}$. Thus, Smolyakovs Rutherford equation takes the form: $\frac{\partial w}{\partial t}=\mu_{*} D_{R}\left(\frac{1}{w}-\frac{1}{w_{0}}-\frac{8 r_{i} \delta \beta_{p}^{*}}{s w^{2}}\right)$, it leads to saturated self-consistent islands.

\section{Snake-like perturbations during LHCD}

\subsection{Suppression of instabilities with LHCD}

Lower-Hybrid Current Drive (LHCD) experiments were carried out in the HL-1M hydrogen plasmas [8]. Up to $0.85 \mathrm{MW}$ of $\mathrm{LH}$ power has been applied to the plasma. The effect of LHCD on central MHD activities [sawtooth $(m=0), m=1$ mode] were studied with the fluctuations observation of the soft X-ray emission.

In figure 7 and 8 , typical experimental results of the central MHD activities during LHCD are shown. Figure 7 presents two soft $\mathrm{x}$-ray signals on which the sawtooth activities were suppressed during LHCD, and time histories of the significant variation in stored energy and internal inductance. The stored

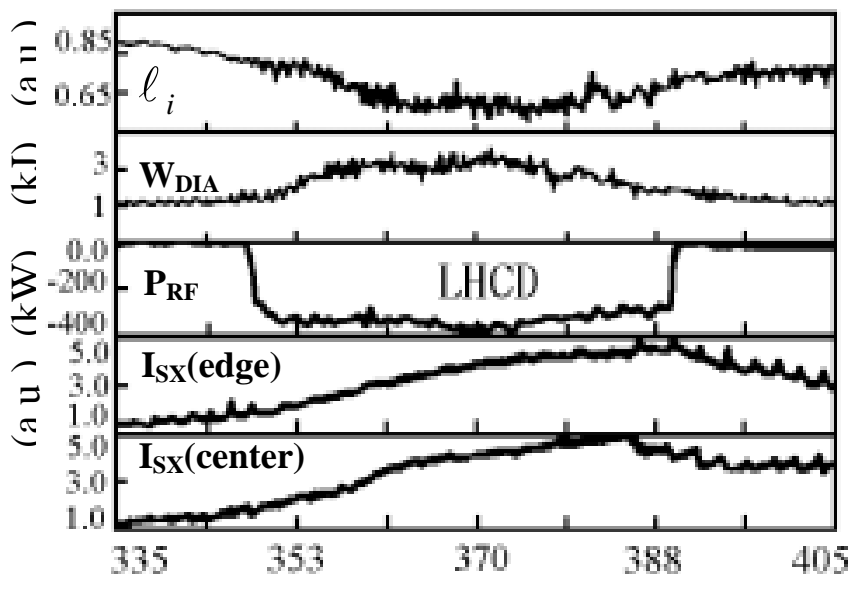

Figure 7. $\ell_{i}, \mathrm{~W}_{\mathrm{DIA}}, \mathrm{P}_{\mathrm{RF}}$, two soft $\mathrm{X}$-ray signals as a function of time for a shot in which suppression of sawtooth activities by RF-power pulse was observed. 
energy measured by the diamagnetic loop rises from $1 \mathrm{~kJ}$ to $3 \mathrm{~kJ}$, and the internal inductance drops from 0.79 to 0.6 during suppression. Figure 8 shows the complete suppression of the $\mathrm{m}=1$ mode by RF-power pulse in another discharge. Strong $\mathrm{m}=1$ mode activity is observed before LHCD, it is suppressed for $\sim 40 \mathrm{~ms}$. During this time interval, the central soft X-ray emission increases gradually, and the line integrated ionic charge state $\mathrm{Z}_{\mathrm{eff}}$ determined from visible bremsstrahlung measures of plasma center, increase from 1.6 to 2.5. In general, there is a threshold value of RF-power for the suppression of sawtooth and $m=1$ mode on HL-1M . However, some shots above these threshold powers did not stabilize and their sawtooth period is enlarged. The increase of double to threefold its initial valve has been observed for the discharges with electron density $\overline{n_{e}}=1.5-1.8 \times 10^{13} \mathrm{~cm}^{-3}$.

The MHD instabilities related to the current profile, and the change in internal inductance $\left(l_{i}\right)$ is consistent with the change in the current profile. The modification of $l_{i}$, which is detected with diamagnetic method, has been observed during sawteeth and $\mathrm{m}=1$ mode suppression in LHCD discharges. Usually, a small decrease in $l_{i}$ during sawteeth and $\mathrm{m}=1$ mode suppression by LHCD has been detected, it suggests that the disappearance of the $q=1$ surface in the plasma and the

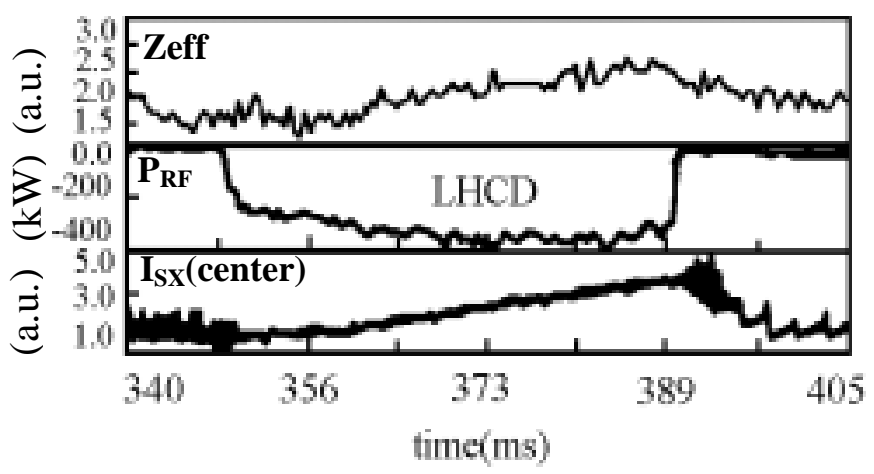

Figure 8. Zeff, $\mathrm{P}_{\mathrm{RF}}$, central soft $\mathrm{X}$-ray signal as a function of time for a shot in which complete suppression of the $\mathrm{m}=1$ mode by RF-power pulse was observed. current profile has flattened.

In some discharges, however, the measured value $l_{i}$ in the stabilized portions is nearly same with the one before RF-pulse.The plasma current profile and q- profile were reconstructed by equilibrium code Efit-T [9] using magnetic probe data and pressure data, the reconstructed results were identical with other diagnostic results, such as the soft x-ray data and displacement measurements. For sawtoothing discharges, the position of the $q=1$ surface determined by the code is in good agreement with the observed sawtooth inversion position. Although the value of $\mathrm{q}_{0}$ cannot be determined with great accuracy, the relative change is more demonstrative. The calculations suggest that the stabilization of sawtooth is caused by the flattening of current profile which leads to the disappearance of the $q=1$ surface in the plasma.

Sometime, the recurrence of sawteeth and/or $m=1$ mode was found at the later phase of RF-pulse in which the sawteeth were suppressed. It suggests that the current profile may be barely stable and that a slight change in the imposed RF current profile may be sufficient to cause central MHD instability. Furthermore, a rotating $m=1$ mode oscillation with snake-like features was frequently observed at the end or later phase of the suppression of sawteeth (or $\mathrm{m}=1$ mode), which will be discussed below.

\subsection{Production of snakes during LHCD}

Snake-like perturbations have been observed in discharges without pellet injection on the JET Tokamak[2], known as spontaneous snake. Similar type of snake-like perturbations was 
observed in LHCD plasmas of HL-1M at the end or later phase of the sawteeth suppression. The snake phenomena observed here is distinctly different from the spontaneous snake found in JET especially in condition for production although there may be similarities in the excitation mechanism via impurity accumulation.

Figure 9 shows temporal evolution of soft X-ray emission signals during LHCD. After quite a long time of sawtooth stabilization (usually 20 to 40ms), snake-like oscillations are observed after LHCD pulse or at late phase of LHCD pulse Usually, snakes are formed accompanying with sawtooth recurrence. They can survive to the sawtooth crashes, and the lifetime is quite long (two or three times of sawtooth periods). Its rotating frequency is about $7 \mathrm{kHz}$, and its minor radius is about $2 \mathrm{~cm}$.So,compared with pellet-induced snakes, snake in LHCD has a much higher rotating frequency but smaller minor radius. To make properties of the snake appear

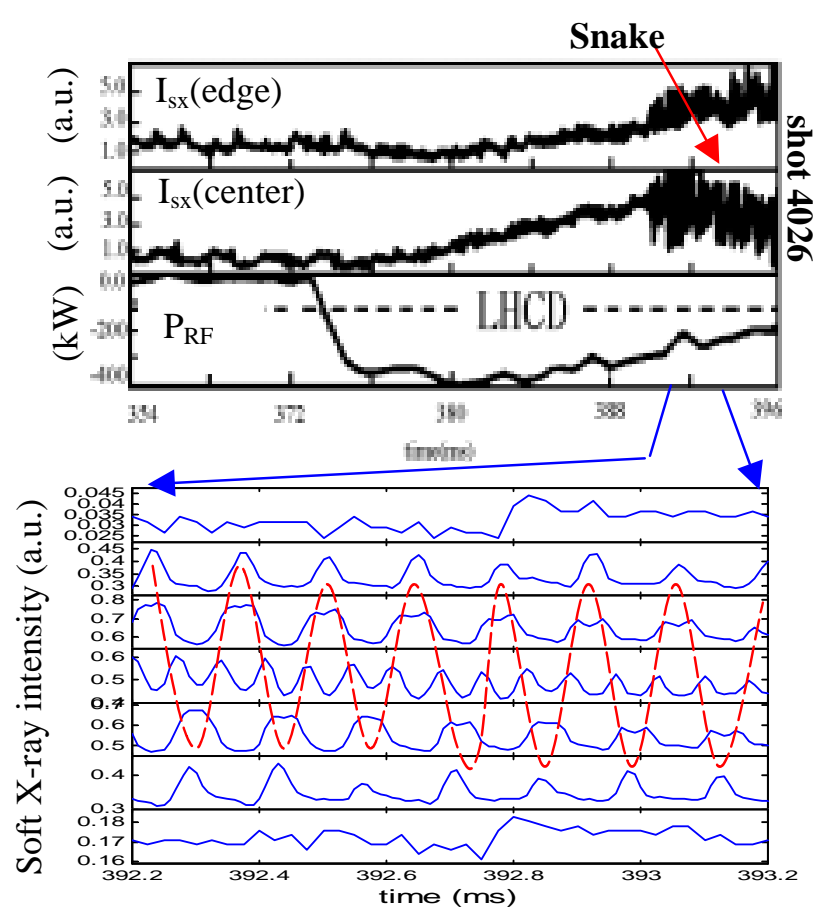

Figure 9. The temporal evolution of Soft X-ray emission signal during LHCD. Snake-like are observed before the onset of sawteeth recurrence (rotating frequency $7 \mathrm{kHz}$ ).

more clearly, we performed SVD to the soft X-ray chord-integrated measurements [10]. The analysis give us a much more clear image of the temple evolution and profile structure of the most important components of the perturbation, namely, sawtooth activity and snake-like perturbation, as shown in Figure 10. The radius of snake is identical to the sawtooth inversion

(a) Temporal components (shot 4026)

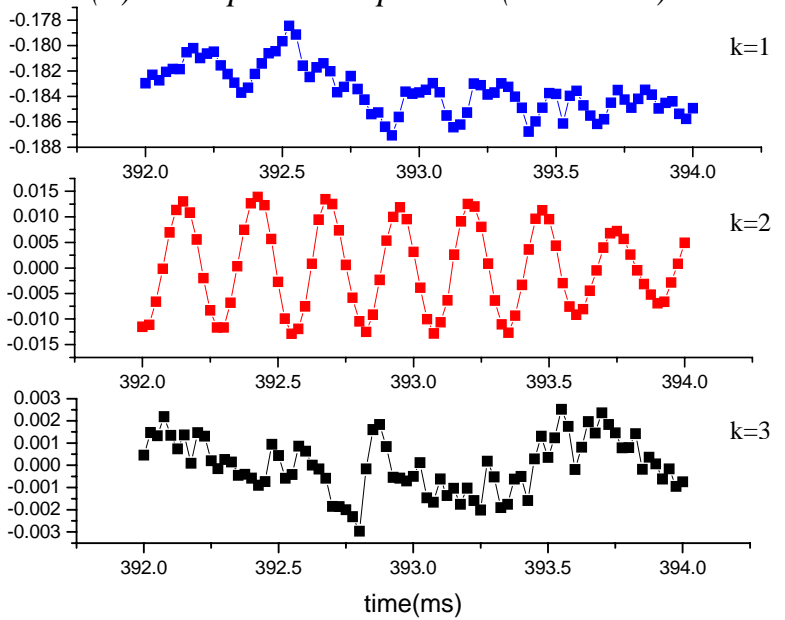

(b) Space-like eigenfunctions

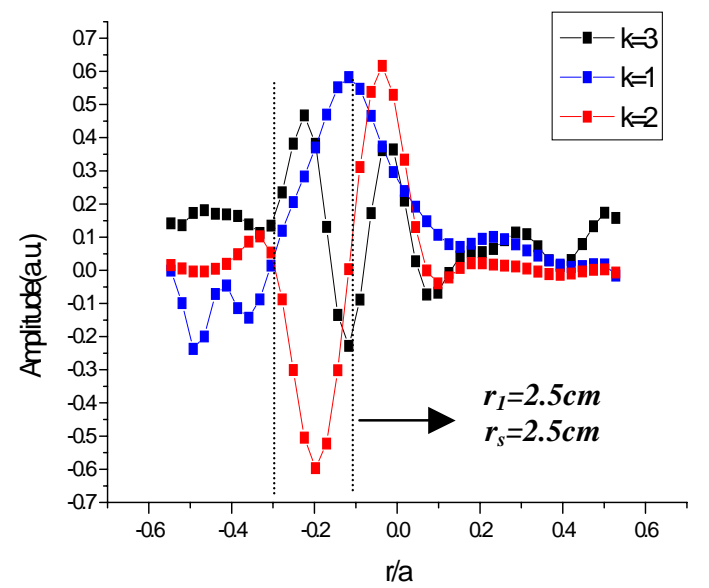

Figure 10. SVD analysis of shot 4026 (a) time evolution of SVD principal components;(b) three spatial eigenfunctions with the largest eigenvalues. The $\mathrm{k}=1$ component corresponds to average intensity in time and in space, $\mathrm{k}=2$ component describes snake-like oscillation $(\mathrm{m}=1$ mode), while $\mathrm{k}=3$ component corresponds to variation due to sawtoothing. 
radius, which is different from the case in pellet injection experiments mentioned above.

Usually, before the onset of sawteeth recurrence after sawtooth stabilization, a normal $\mathrm{m}=1$ MHD activity can be observed. There is a probability of $30 \%$ that the normal $\mathrm{m}=1$ activity can evolve to a snake oscillation. The fact that snakes are only found to occur at the end or later phase of the suppression of sawteeth (or $m=1$ mode) by LHCD implies the formation of snakes-like perturbation is related to the instabilities suppression.

\subsection{Interpretation to snake observed during LHCD}

In these LHCD discharges, after a long period of suppression of instabilities a peaked soft $\mathrm{X}$-ray (SXR) has been observed, and the increase of impurities radiation detected is distinct. This is more probably due to the fact that the beneficial effects of sawtooth activities (preventing the central accumulation of impurities) have lost during the sawtooth suppression phase. Usually there is an increase in average effective charge $Z_{\text {eff }}$ obtained from quantitative analysis of the visible bremsstrahlung emission at a wavelength around $5360 \stackrel{0}{A}$ as shown in

Fig 8. Ultra-Violet (VUV) spectrometer and centrally radiated power measurement with Bolometers also indicate that the impurities are enhanced, while the electron density obtained shows a slight increase but no significant peaking during central MHD activities stabilization and there is minor temperature increase. In JET, spontaneous snakes have also been observed in discharges exhibiting impurity accumulation at its early times. Since the discharges with snakes during LHCD show clear evidence for impurity accumulation for a lone time during sawtooth suppression, it is probable that the same mechanism for the formation of spontaneous snake in JET can be used to explain the excitation of snake during LHCD. The contribution of impurity effect to the stability in terms of $\Delta^{\prime}$, the discontinuity of the vector potential amplitude across the resonant layer, is given by [3] $\Delta_{\eta}^{\prime}=\frac{128}{15 \pi} \frac{2-s}{s} \frac{\delta Z_{\text {eff }}}{Z_{\text {eff }}} \frac{1}{w_{s}}$. So, it can be drawn that the impurity effect is destabilizing. It seems that the accumulation of impurities at plasma center during stabilization could leads to the formation of an $m=1$ island with lifetime exceeding the global resistive diffusion time. It indicated that the good performance with peaking profiles after LHCD is limited by snake instability, along with sawtooth instability.

\section{Conclusion}

Snake phenomena are observed in HL-1M after pellet injection for a large range of density under certain conditions related to the penetration depth. Location of snake oscillation and its relationship to $\mathrm{q}=1$ surface have been analyzed from multi-channel soft $\mathrm{X}$-ray and $\mathrm{H}_{\alpha}$ measurements. Quantitative analysis of the experimental results indicated that, when the plasma initial q profile is quite flat, pellet injection may cause the rational $\mathrm{q}=1$ surface shift inside to a inner region (where a large amount of particles are deposited) because of changes of local temperature and density, thus a magnetic island that traps particles released from the pellet is formed leading to a snake oscillation.

Snake-like perturbation is also observed in LHCD plasmas of HL-1M at the end or later phase of the sawteeth suppression. Its condition for formation is related to sawtooth stabilization for a long period with impurity accumulated at plasma center. It indicated that the good performance with peaking profiles after LHCD is limited by snake instability, along with sawtooth instability. 
Snake-like phenomena may occur either in consequence of pellet injection or if the onset of sawtooth is delayed by LHCD. There are evidences that both the effects of bootstrap current and impurity play important roles in the formation of snake oscillation. In the pellet -induced case, the bootstrap current term dominates over the impurity term. On the other hand, the main contribution comes from impurity term in the LHCD induced case. Further experiments are needed to estimate the current profile modification after pellet injection or application of LHCD. In addition, direct measurements of the perturbations of electron temperature and electron density in the snake region are in the plan on HL-2A device. The mechanism of snake-like perturbations will be further studied.

\section{Reference}

[1] A.Weller,at.al,.Phys.Rew.Lett.59,2303 (1987).

[2] Y.Kamada, T.Ozeki, and M.Azumi, Phys.Fluids B 4,124 (1991).

[3] A.L.Pecquet at al., Nucl. Fusion 37, 451 (1997).

[4] R.D.Gill, A.W.Edwards,D.Pasini A,Weller, Nucl. Fusion, 29 (1992) 723.

[5]Longwen Yan, et al,. Nucl. Fusion 42 (2002) 265.

[6]W.Park,D.A.Monticello, and T.K.Chu,Phys.Fluids 30(1987)285.

[7]A.Thyagaraja and F.A.Haas,Phys.Fluids B3(3),580(1991).

[8]E.Y.Wang,"Recent Experiments and Confinement Studies in the HL-1M

Tokamak"Sixteenth IAEA Fusion Energy Conference, Montreal, Canada, 7-11 October 1996.

[9] Xu,W.B,Chin.Phys.Lett.16, 114 (1999).

[10] G.Fuchs,Y. Miura and M.Mori.,Plasma.Phys.Control.Fusion 36 ,307(1994). 